\title{
Statistical Models for Mobile Telephony Growth in Oman
}

\author{
${ }^{*}$ Ahmad, M. I., Alsaadi, M. K, Almamri, A. \\ Sultan Qaboos University, Muscat, Oman \\ *idrees@squ.edu.om
}

\begin{abstract}
This study examines the growth of mobile telephony in Oman and investigates the determinants of this growth. The growth rates were estimated by fitting four statistical models namely, Logistic, Gompertz, Exponential and Autoregressive to fifteen years of data on annual numbers of mobile subscribers. The in-sample and out-of-sample forecasting performance of these models was assessed by Mean Absolute Deviation (MAD) and Mean Square Error measures. Based on this criterion it was observed that the Gompertz model outperformed the other models under consideration and gave the minimum forecast error. This model was then used to estimate the speed of diffusion which was regressed on potential factors that could affect the growth of mobile telephony. The factors considered were fixed line service, prepaid service, per capita income, consumer price index and the number of operators. All these factors were found to have significant effect on the speed of diffusion of the mobile telephony in Oman.
\end{abstract}

Keywords: Growth models, speed of diffusion, forecasting, mobile telephony

\section{Introduction}

Background: Telecommunications infrastructure is essential to national competitiveness and closely related to economic growth (Roller \& Waverman, 2001). Telecommunications technology has evolved from wireline to wireless, so it is resulted in better connectivity and easy accessibility among people in today's dynamic mode of life. The telecom industry will certainly become the key of industry in the future. As telecom market had been fully liberalized, globalization and fleetly advanced in technology, the innovations of technology, instantiate Fax, Mobile phone, Fiber cable, Internet telephony and etc, changed the life-style of people in recently decade. We can anticipate that advance of telecom industry will become more diversified and complicated to change original structure of telecom industry. The need for information to be available anywhere and anytime has been a driving force for advances in the wireless communication technology to diffusing around the planet faster than any other communication technology to date. One of an innovation wireless communications technology is Mobile (cellular) telephony technology which is offers both benefits and novelty to users. Mobile telephony grew rapidly in the $1990 \mathrm{~s}$ as a result of gradually decreasing service charges. The diffusion of Mobile telephony is extremely important to economic growth in developing countries. To help Decision-makers in our country to have a clear picture about this Technical phenomenon, we decided to study this phenomenon by using technological forecasting such as growth curves and regression analysis.

The S-shape curves: The S-shape curves describe many natural phenomena and also used for technological forecasting known as growth curves. There are more than 100 logistic curves usually based on the logistic function. They start slowly, followed by a steep growth, and then slowing down approaching asymptotically the limit. The S-shape is caused by the fact that the innovation - whether it's a technology or a pathogen - has first to come in from outside the social system, and that means relatively few people are susceptible to begin with. Once the innovation is established within the system, more and more people come into contact with it, and the rate of spread increases. Eventually, so many members of the community have been affected (adopted the innovation) that the system runs out of unaffected members, and the rate of spread slows and eventually stops.

Oman Diffusion of Mobile Telephony: Information technology and communications have now become the main elements that move forward and help Oman in its national development process. The number of mobile holders increased substantially since the launch of these services in 1996. In graph 2 we can see how the number of subscribers going on. 


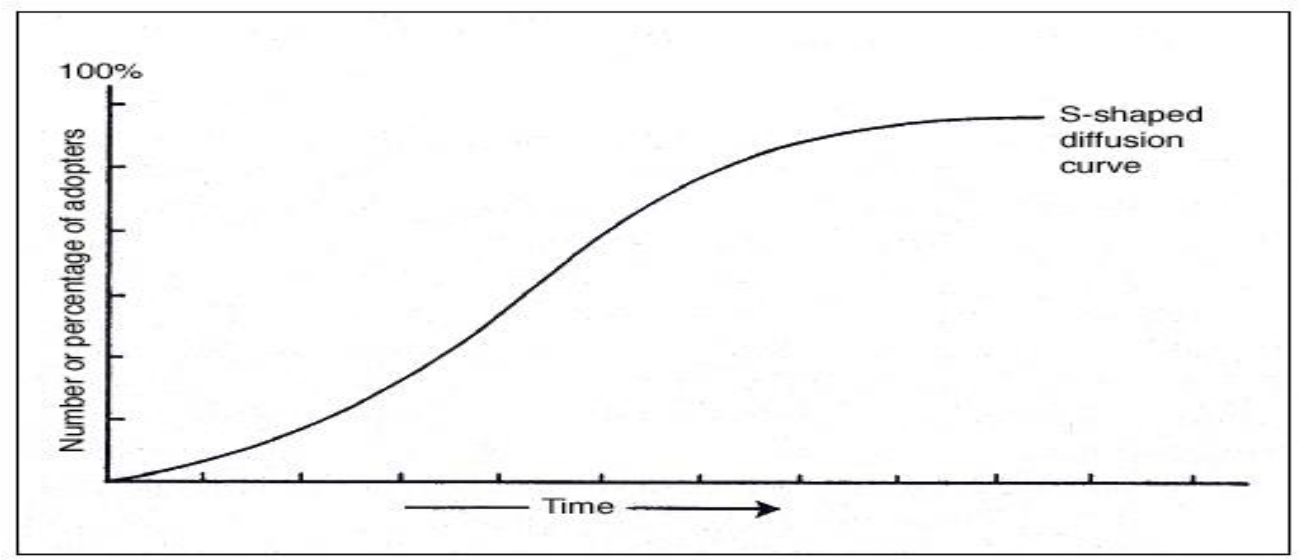

Figure 2: Number of mobile telephony subscribers in Oman (1996-2010)

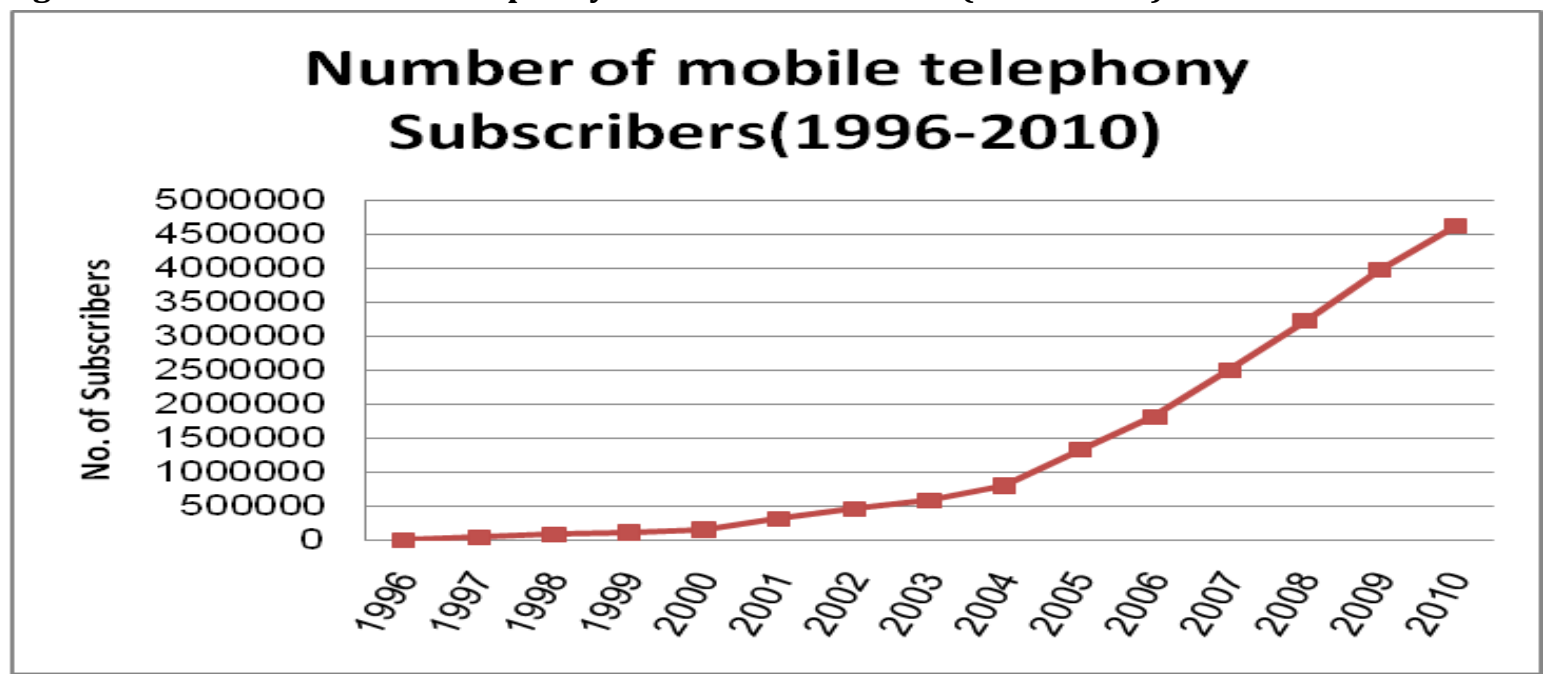

The graph shows a rapid increase in 2001because prepaid mobile services and SMS were introduced (Naqvi \& Al-Shihi, 2009). Also another sharp rise occurs in 2005 because Nawras became a second provider with Oman-Tel and the prices were fixed during the same period of time. Growth in mobile telephony is significant in unsaturated developing countries (i.e. Oman and Qatar), and relatively slower in near-saturated developed countries (Japan).

Figure 3: growth of mobile telephony penetration

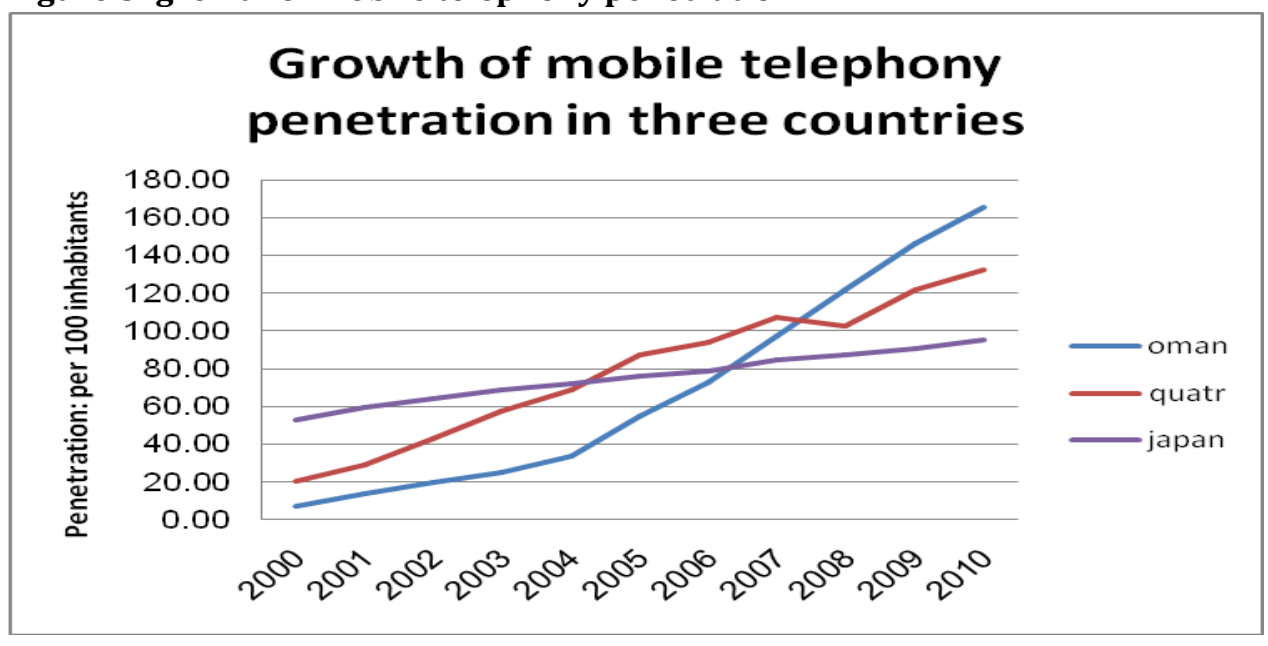


From graph 3 we see Oman has sharply increase in the penetration rate, especially in years 2001and 2005 that because policy changing was made.

Source of Data: The study is based on annual national data. The data on the number of subscribers for the period 1996-2010 is taken from annual reports of Telecommunication Regulatory Authority and International Telecommunications Union (World Telecommunications Indicators).(http://www.itu.int). Also, we take Data on GDP/capita and CPI from annual reports of ministry national economic. (http://www.ncsi.gov.om).

\section{Methodology}

Statistical model: There are two types of models which are linear and non-linear. A linear regression model provider a large and rich framework that suits the needs of many analysts. However, linear regression cannot be adequate for all problems, because sometimes the response and the predictors are related through a known nonlinear function. The basic idea of nonlinear regression is the same as that of linear regression, namely to relate a response $\mathrm{Y}$ to a vector of predictor variables $\mathrm{x}=\left(\mathrm{x}_{1}, \ldots, \mathrm{x}_{\mathrm{k}}\right)^{\mathrm{T}}$. Nonlinear regression is characterized by the fact that the prediction equation depends nonlinearly on one or more unknown parameters. Whereas linear regression is often used for building a purely empirical model, nonlinear regression usually arises when there are physical reasons for believing that the relationship between the response and the predictors follows a particular functional Form.

The normal linear regression model may be written

$y_{\mathrm{i}}=\mathrm{x}_{\mathrm{i}}^{\prime} \beta+\epsilon_{\mathrm{i}}$

Where $\mathrm{x}_{\mathrm{i}}^{J}$ is a (row) vector of predictors for the $\mathrm{i}^{\text {th }}$ of $\mathrm{n}$ observations, usually with a 1 in the first position representing the regression constant; $\beta$ is the vector of regression parameters to be estimated; and $\epsilon_{\mathrm{i}}$ is a random error, assumed to be normally distributed, independently of the errors for other observations, with expectation 0 and constant variance: $\varepsilon_{\mathrm{i}} \sim \mathrm{NID}\left(0, \sigma^{2}\right)$.

Diffusion Models Applying a growth model analogy is the first step in analyzing mobile telephony diffusion; the Logistic, Gompertz, Growth model and autoregressive (AR) model have their own principles and advantages. Those models have been briefly reviewed by (Xielin, Feng-Shang \& Wen-Lin, 2012) ${ }^{3}$ and are given as follows:

1) Logistic Model: The Logistic model is expressed as

$$
\frac{\mathrm{dN}}{\mathrm{dt}}=\mathrm{rN}\left(1-\frac{\mathrm{N}}{\mathrm{K}}\right) \text { or } \frac{\mathrm{dN} / \mathrm{dt}}{\mathrm{K}-\mathrm{N}}=\mathrm{r} \frac{\mathrm{N}}{\mathrm{K}}
$$

Where $\mathrm{N}$ is the number of adopters at time $\mathrm{t}, \mathrm{r}$ is the intrinsic growth rate, and $\mathrm{K}$ is the number of adopters at equilibrium, namely, the maximum number of adopters. The solution to this first-order differential equation is

$$
\mathrm{N}(\mathrm{t})=\frac{\mathrm{K}}{1+\mathrm{e}^{-\mathrm{r}_{\mathrm{T}}\left(\mathrm{T}-\mathrm{m}_{\mathrm{T}}\right)}}
$$

2) Gompertz Model: The Gompertz model is represented as

$$
\frac{\mathrm{dN}}{\mathrm{dt}}=\mathrm{rN} \ln \frac{\mathrm{K}}{\mathrm{N}} \text { or } \frac{\mathrm{dN} / \mathrm{dt}}{\mathrm{N}}=\mathrm{r} \ln \frac{\mathrm{K}}{\mathrm{N}}
$$

Where $\mathrm{N}$ is the number of adopters at time $\mathrm{t}, \mathrm{r}$ is the intrinsic growth rate, and $\mathrm{K}$ is the number of adopters at equilibrium, i.e. maximum number of adopters. The solution to this first-order differential equation is

$\mathrm{N}(\mathrm{t})=\mathrm{Ke}^{-\mathrm{r}(\mathrm{t}-\mathrm{m})}$

3) Growth curve Model: the Growth curve model is represented as:

$y_{\mathrm{t}}=\beta_{0}\left(\beta_{1}^{\mathrm{t}}\right) \varepsilon_{\mathrm{t}}$

Then we take nature Log both side 


$$
\ln y_{t}=\ln \beta_{0}+\mathrm{t} \ln \beta_{1}
$$

To get prediction values for Growth model we must transform such nonlinear model to one that is linear in the parameter. The model:

$$
y_{\mathrm{t}}=\beta_{0}\left(\beta_{1}^{\mathrm{t}}\right) \varepsilon_{\mathrm{t}}
$$

Can be transformed to linear model by taking logarithms on both sides.

$$
\ln y_{\mathrm{t}}=\ln \beta_{0}+\mathrm{t} \ln \beta_{1}+\ln \varepsilon_{\mathrm{t}}
$$

If we let $\alpha_{0}=\ln \beta_{0}, \alpha_{1}=\ln \beta_{1}$ and $\mu_{\mathrm{t}}=\ln \varepsilon_{\mathrm{t}}$

Then the transformed version of the model becomes

$\ln y_{\mathrm{t}}=\alpha_{0}+\alpha_{1} \mathrm{t}+\mu_{\mathrm{t}}$

4) Autoregressive AR (1) model: We use first Autoregressive, the formula of that is below:

$$
y_{\mathrm{t}}=\beta_{0}+\beta_{1} * y_{\mathrm{t}-1}+\varepsilon_{\mathrm{t}}
$$

We find the parameters from OLS method. Actually we use MINITAB to help us to find that parameters. Minitab $^{\circledR}$ Statistical Software (2014).

choice of the model: There are several factors and steps involved to choose of a suitable statistical model among several very closely competing ones, the most important of which are, estimation of the parameters of the model and tests of goodness of fit. We used non-linear numerical optimization for Logistic and Gompertz models while the Ordinary Least Square (OLS) method of estimation was adopted for Growth curve and Autoregressive models. The in-sample and out-of-sample forecasting performance of these models was assessed by Mean Absolute Deviation (MAD) and Mean Square Error measures.

Determinants of Diffusion Rate :After the best diffusion model was identified, the determinants of mobile telephony diffusion rate were estimated. We had chosen six items as independent variables. The variables are Fixed-line telephone subscribers (FIX), Prepaid service (PP), Per capita GDP (GDP), Consumer price index (CPI) and Number of operators (OPR). Those variables we thought it have an effect on the speed of diffusion. So, by using linear regression with $\boldsymbol{r}_{\boldsymbol{t}}$ (as response) and the others (as predicting) then we can say what is impact of that factors on the speed of diffusion.

Thus, the relationship of the independent factors with the diffusion rate is:

$\boldsymbol{r}_{t}=\boldsymbol{\beta}_{0}+\boldsymbol{\beta}_{1} F I X+\boldsymbol{\beta}_{2} P P+\boldsymbol{\beta}_{3} G D P+\boldsymbol{\beta}_{4} C P I+\boldsymbol{\beta}_{5} O P R$

Fixed-line telephone subscribers (FIX): it represent the number of fixed-line phones subscribers. This factor may help us to judge whether mobile phones complement or are substitutes for fixed-line phones. If the coefficient of FIX, i.e., $\beta 1$, is positive, then fixed-line and mobile phones are complementary; however, if the coefficient is negative, they are substitutes. Prepaid service (PP): we use a dummy variable to represent availability of this service in Oman among year of providing this service. By the way this service introduces in 2001. A PP value of 0 indicates before 2001, the year in which PP was launched, and a PP value of 1 indicates since 2001. Per capita GDP (GDP): Gross domestic product was used as an indication of national economic health. Consumer price index (CPI): we take price for telecommunication and try to see if it's well effect the diffusion rate. Number of operators (OPR): The OPR correlates strongly with intensification of competition and mobile telephony diffusion. An increasing OPR implies intensified competition and, hence, rapid mobile telephony diffusion. Oman Mobile was the only mobile telephone operator in Oman until Nawras entered in 2005.

\section{Analysis}

This study empirically investigates past and present patterns of mobile telephony diffusion in Oman using an appropriate diffusion model, with a particular focus on diffusion drivers. To eliminate ad hoc model selection, this started adopts the nonlinear least squares procedure and compares the fitness and forecasting accuracy of four conventional models, namely, the Logistic, Gompertz, growth ,AR(1) models, by having data on an annual basis during 1996-2009 to identify which of the four models is best in both fitting and forecasting. The data is divided into two periods-estimation period (year1996 to year2009) and forecasting period (year2010). After the best model founded, then apply the selected model to 
identify diffusion rate. All four models were tested with data, which is divided into two periodsestimation period (year 1996 to year 2009) and forecast period (year 2010).

The estimation results of all the models: From the table 1 below it's clear that, Gompertzs model is the best in fitting data. Also, same model is good for forecasting.

Table1: Estimation results for all models

\begin{tabular}{lllll}
\hline Parameter & Logistics & Gompertzs & Growth model & AR(1) \\
\hline b1 & 7080000 & 42420000 & 2.3 & 77995 \\
b2 & 13.43 & 9.072 & -0.93 & 1.26 \\
b3 & -0.4349 & 0.09629 & - & - \\
Residual standard error & 40450 & 59100 & 0.09 & 1154391615 \\
Fitted MAD(1996-2009) & 5004572 & 41369.20 & $194,390.95$ & 76401.93 \\
Forecasted MAD(2010) & 2227335.15 & 384391.14 & $3,515,160.52$ & $681,174.50$ \\
\hline
\end{tabular}

Now, we have to find the diffusion rate from Gompertzs model (Meade \& Islam, 2001).

Now, we have to find the diffusion rate from Gompertzs model:

$$
\frac{\mathrm{dN}}{\mathrm{dt}}=\mathrm{rN} \ln \frac{\mathrm{K}}{\mathrm{N}} \text { or we can put it in form of } \frac{\mathrm{dN} / \mathrm{dt}}{\mathrm{N}}=\mathbf{r} \ln \frac{\mathrm{K}}{\mathrm{N}}
$$

We know that $\mathrm{dN} / \mathrm{dt}$ it's same as $\mathrm{N}_{\mathrm{t}+1}-\mathrm{N}_{\mathrm{t}}$, so

$$
\frac{\mathrm{N}_{\mathrm{t}}-\mathrm{N}_{\mathrm{t}-1}}{\mathrm{~N}_{\mathrm{t}-1}}=\mathrm{r} \ln \frac{\mathrm{K}}{\mathrm{N}_{\mathrm{t}-1}}
$$

also we can re-write $\mathrm{N}_{\mathrm{t}+1}-\mathrm{N}_{\mathrm{t}}$ as this equality formula $\mathrm{N}_{\mathrm{t}}-\mathrm{N}_{\mathrm{t}-1}$

$\frac{\mathrm{N}_{\mathrm{t}+1}-\mathrm{N}_{\mathrm{t}}}{\mathrm{N}_{\mathrm{t}}}=\mathrm{r} \ln \frac{\mathrm{K}}{\mathrm{N}_{\mathrm{t}}}$

Finally, we can re-write (let $\mathrm{K}$ as $\overline{\mathrm{N}}$,its saturation level)

$r_{t}=\frac{N_{t}-N_{t-1}}{N_{t-1}\left(\ln \bar{N}-\ln N_{t-1}\right)}$

Determinants of Diffusion Rate : Mobile telephony diffusion studies focused on determinants of diffusion growth rate, i.e., the diffusion rate e. g Massini (2003). The Gompertzs diffusion model is selected for studying the factor affecting the speed of diffusion .the speed varying Gompertzs diffusion model is described:

$$
r_{t}=\frac{N_{t}-N_{t-1}}{N_{t-1}\left(\ln \bar{N}-\ln N_{t-1}\right)}
$$

Where,

$\mathrm{N}_{\mathrm{t}}$ is the number of mobile subscribers at time $\mathrm{t}$.

$N_{t-1}$ is the number of mobile subscribers at time t-1.

$\bar{N}$ is the saturation level.

$r_{t}$ is the measure diffusion speed (co-efficient of imitation)

We can see from Table 2, the estimation results for determinants are shown also we put near each determinates the p-value.

Table 2: ESTIMATION RESULTS FOR DETERMINANTS OF DIFFUSION RATE OF MOBILE

\begin{tabular}{lllllll}
\hline & Constant & FIX & $\boldsymbol{P P}$ & $\boldsymbol{G D P}$ & $\boldsymbol{C P I}$ & OPR \\
\hline coefficient & 2.43 & -0.0000092 & 0.15 & 0.000024 & -0.0067 & 0.18 \\
p-value & $0.043^{\mathrm{a}}$ & $0.015^{\mathrm{a}}$ & $0.07^{\mathrm{b}}$ & $0.079^{\mathrm{b}}$ & 0.25 & $0.068^{\mathrm{b}}$ \\
\hline
\end{tabular}

\footnotetext{
$\mathrm{R}-\mathrm{Sq}=63.3 \%$

a...this significant at $5 \%$

b....this significant at $10 \%$
} 
Durbin-Watson statistic $=2.42$, so there is no autocorrelation

Table 2 lists the estimation results for growth rate determinants. Estimation results demonstrate that FIX, PP, GDP, and OPR were significant, while, CPI were insignificant. We can read more from previous model:

1) Fixed-Line Telephone Subscribers (FIX): As the FIX coefficient is negative, this study demonstrates that mobile telephony is substitutes to fixed-line telephony in Oman-developing country. This empirical finding support the general assumption that mobile telephony is complementary to fixed-line telephony in developed countries and substitutive in developing countries International Telecommunications Union (ITU)(1999).

2) Prepaid Service (PP): since the PP is significance, so it has an effect on speed of diffusion rate. This services introduced in 2001.

3) Per capita GDP (GDP): this factor has significant effect on the rate of diffusion. So as we have healthy economic that well help to speed up the rate of diffusion.

4) Consumer price index (CPI): this shows that the inflation will not stop customers to buy telephone. This factor has not significant effect on increase the diffusion rate.

Number of operators (OPR): the number of service providers effect $18 \%$ of diffusion rate. This factor has significant effect to increase the diffusion rate.

Saturation level: Saturation level is the period when the S-curve start to decrease, we found the it by calculate the difference between actual data(diff.y $\left.=y_{t}-y_{t+1}\right)$ and also found the difference between fitted data of Gompertz model(diff.gomp $=y_{t}-y_{t+1}$ ). Then we plot the difference of actual data versus difference of fitted data of Gompertz model. Graph 2 shows that the saturation level will be in year 2019 (so, it means that the number of subscribers well start to decrease in year 2019).

Graph 2: saturation level

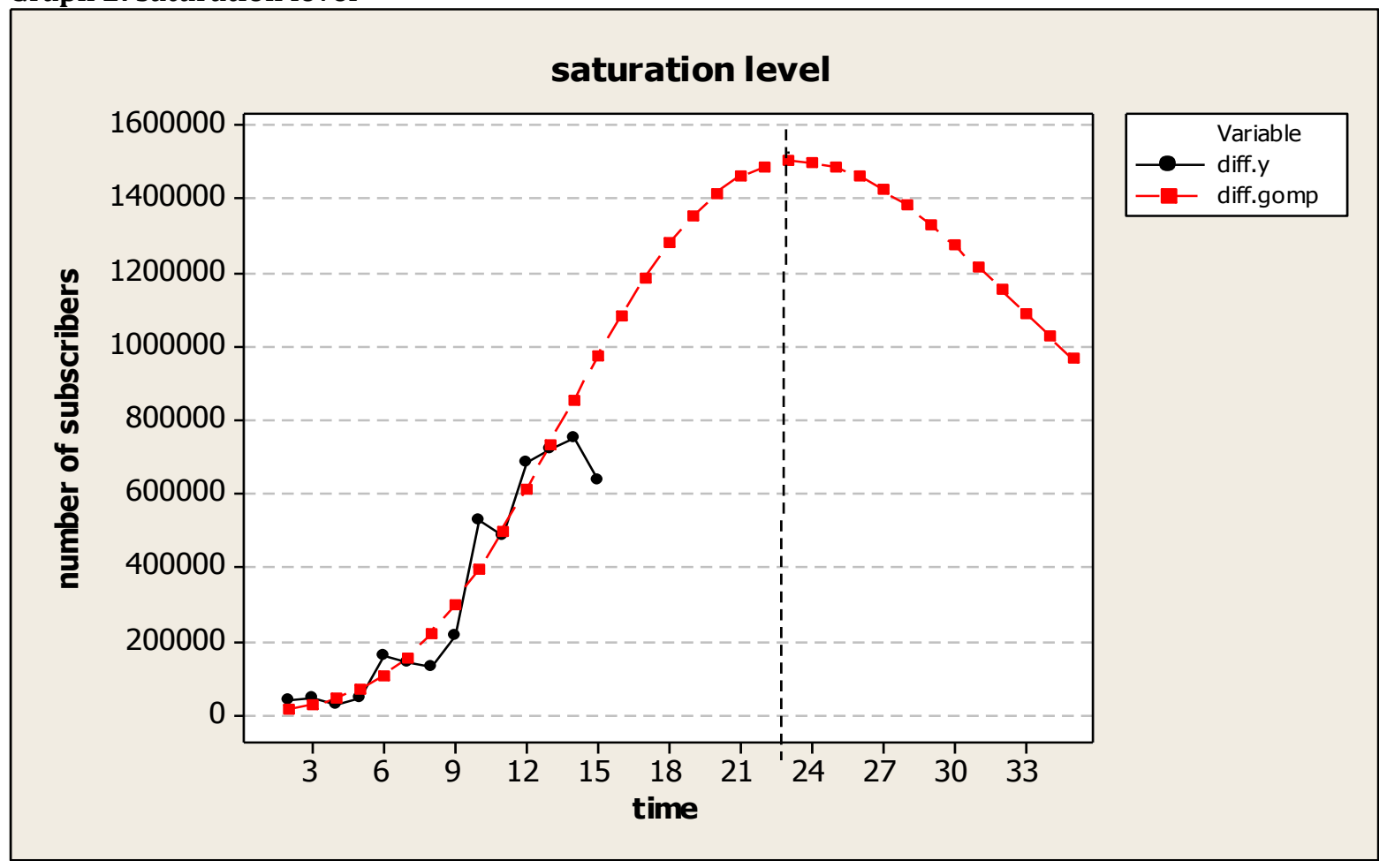

\section{Conclusion}

Mobile telephony diffusion is an extremely important to economic growth in developing countries. To help Decision-makers in our country to have a clear picture about this Technical phenomenon, we decided to study this phenomenon by using the most appropriate model and determinants, and also forecast its future growth. Based on the epidemic theory of innovation diffusion and country-specific studies on diffusion of mobile telephony, four models were applied for studying the diffusion of mobile 
telephony in Oman. After analysis, we find the Gompertzs model adequately describes the path of mobile telephony diffusion in our Country. Then we study some factors that may have impacted the diffusion speed of mobile telephony. We choose number of Fixed-line telephone subscribers (FIX), prepaid service (PP), Per capita GDP, Consumer price index (CPI), and Number of operators (OPR) as the determinant of growth. Empirical results indicate that the four main determinants of diffusion FIX, PP, GDP, and OPR were significant, while, CPI were insignificant.

We can say that Competition amongst service providers came out as the key factor responsible for the diffusion speed in Oman. Also, the fixed line telephony in our country was found to have a negative effect on mobile telephony diffusion speed. It implies that the adopters in Oman have considered mobile telephony as a substitute to fixed line telephony. We found, Oman will reach the saturation level of subscribers in year 2019; this is as result shown by graph of Gompertzs model. The diffusion of mobile telephony in Oman and the results derived from this study suggest that both efficiency (service provider) and rationality (government intervention) are embedded in the Oman telecommunication sector. The findings of this study can be used by the decision makers (government and service providers) when evaluating the investment in fixed line telephony and/or mobile telephony. These findings can also be used in designing the innovative measures for the diffusion of upcoming mobile services.

\section{References}

Frank, L. D. (2004). An analysis of the economic situation on modeling and forecasting the diffusion of wireless communications in Finland. Technol. Forecasting Soc. Change, 71, 391-403.

International Telecommunications Union (ITU). (1999). World Telecommunication Development Report 1999. Mobile Cellular. Executive Summary. Geneva Switzerland: ITU, 1999.

Meade, N. \& Islam, T. (2001). Forecasting the diffusion of innovations: Implications for time series extrapolation. Principles of Forecasting: A Handbook for Researchers and Practitioners, J. S. Armstrong, Ed. Norwell, MA: Kluwer, 2001.

Massini, S. (2003). The diffusion of mobile telephony in Italy and the UK: an empirical investigation. Economics of Innovation and New Technology, 13(3), 251-277.

Minitab ${ }^{\circledR}$ Statistical Software. (2014). URL: www. minitab.com.

Naqvi, S. J. \& Al-Shihi, H. (2009). M-Government Services Initiatives in Oman. Issues in Informing Science and Information Technology, 6, 817-824.

Roller, L. H. \& Waverman, L. (2001). Telecommunications infrastructure and economic development: A simultaneous approach, Amer. Econ. Rev., 91, 909-923.

Xielin, L., Feng-Shang, W. \& Wen-Lin C. (2012). Diffusion of Mobile Telephony in China: Drivers and Forecasts. IEEE Trans. On Eng. Mang., 59(2), 299-309. 